\title{
Rapid Green Synthetic Protocol for Novel Trimetallic Nanoparticles
}

\author{
B. Karthikeyan and B. Loganathan \\ Department of Chemistry, Annamalai University, Annamalai Nagar, Tamil Nadu 608 002, India \\ Correspondence should be addressed to B. Karthikeyan; bkarthi_au@yahoo.com
}

Received 31 December 2012; Accepted 16 January 2013

Academic Editor: Hamed Bahmanpour

Copyright (C) 2013 B. Karthikeyan and B. Loganathan. This is an open access article distributed under the Creative Commons Attribution License, which permits unrestricted use, distribution, and reproduction in any medium, provided the original work is properly cited.

\begin{abstract}
Metallic nanocomposites and nanotubes can be rapidly prepared under microwave irradiation (MW) from an aqueous solution of metallic precursors in the presence of trisodium citrate as a reductant. For the nanotubes nanoparticles are stabilized by poly $(\mathrm{N}$ vinyl-2-pyrrolidone) (PVP), a protecting agent. PVP is a typical capping and structure-directing agent used for the synthesis of various metallic nanostructures. In this work, we have demonstrated for the first time an MW irradiation approach for the synthesis of trimetallic nanocomposites and nanotubes. The resulting nanohybrids were characterized by UV-Vis spectroscopic analysis, high-resolution scanning electron microscopy (HR-SEM), energy dispersive X-ray spectroscopic analysis (EDX), and Xray diffractometer $(\mathrm{XRD})$ techniques.
\end{abstract}

\section{Introduction}

Over the past decade there has been an increased emphasis on the topic of green chemistry and chemical processes [1]. Colloidal metallic nanoparticles are emerging as an important family of multifunctional nanoscale materials that assist diverse applications in various fields [2-7]. For these essential applications, a variety of preparation routes have been reported for the syntheses of metallic nanosized particles. In order to increase the efficiency of Pt-based bimetallic and trimetallic nanoparticles catalysts have been prepared [8, 9]. Because, in usual metal catalysts, addition of other element can often improve the catalytic activity and selectivity, from the same viewpoint bimetallic and trimetallic nanoparticles are also often investigated. Trimetallic nanoparticles retain an ever greater degree of catalytic activity than the bimetallic and monometallic ones. However, there have been only few reports of trimetallic nanoparticles in the literature. The reported trimetallic nanoparticles were synthesized by only thermal heating method [10-14].

On the other hand, microwave (MW) dielectric heating is fast emerging as a widely accepted rapid processing technology for a variety of synthesis of nanoparticles. In the MW-assisted syntheses of nanoparticles, we found that
MW heating is a rapid technology and it can control the size distribution of the nanoparticles than the conventional heating by thermal convection [15].

In the present work on the preparation of nanosized trimetallic nanocomposites and trimetallic nanotubes from an aqueous solution of corresponding metallic precursors, we have employed trisodium citrate as a reducing agent for the preparation of different combinations of trimetallic nanoparticles and poly(N-vinyl-2-pyrrolidone) (PVP) as a stabilizing agent. Using MW irradiation as a heating source, the rapid, homogeneous, and selective heating in the reaction system at the molecular level for rapid and size-controlled preparation of nanosized particles, which has never been achieved by other conventional methods is reported for the first time.

\section{Materials and Methods}

2.1. Materials. Hydrogen tetrachloroaurate (III) trihydrate $\left(\mathrm{HAuCl}_{4} \cdot 3 \mathrm{H}_{2} \mathrm{O}\right)$, hydrated hexachloroplatinic (IV) acid $\left(\mathrm{H}_{2} \mathrm{PtCl}_{6} \cdot 6 \mathrm{H}_{2} \mathrm{O}\right)$, silver nitrate $\left(\mathrm{AgNO}_{3}\right)$, Palladium chloride $\left(\mathrm{PdCl}_{2}\right)$ trisodium citrate $\left(\mathrm{C}_{6} \mathrm{H}_{5} \mathrm{Na}_{3} \mathrm{O}_{7} \cdot 2 \mathrm{H}_{2} \mathrm{O}\right)$, poly $(\mathrm{N}-$ vinyl-2-pyrrolidone) (PVP; $\left.K=30, M_{W}=40000\right)$, and 


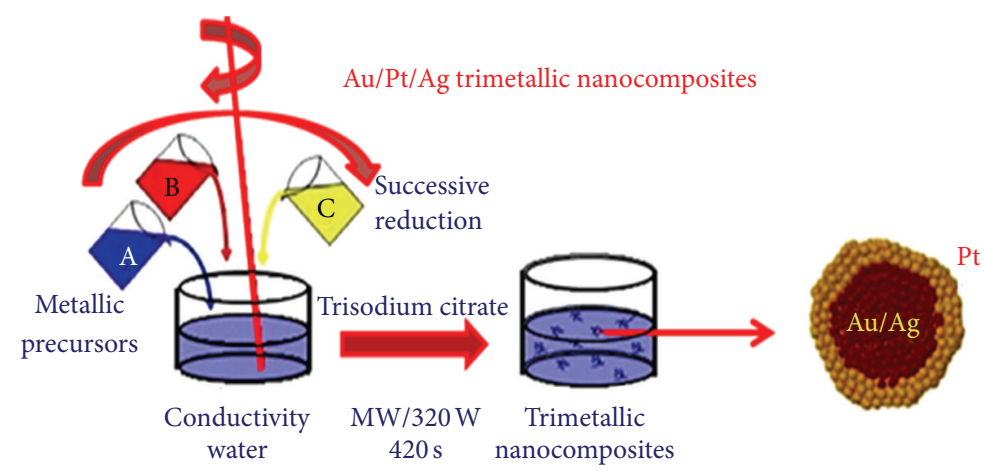

FIGURE 1: MW irradiation method for the preparation of Au-Pt-Ag trimetallic nanoparticles.

all other chemicals used were of analytical grade and the chemicals were purchased from HiMedia Laboratories Pvt. Ltd. (Mumbai, India). All chemicals were used as received without further purification. Conductivity water was used in all experiments.

2.2. Synthesis of Trimetallic Nanoparticles. The Au-Pt-Ag trimetallic nanocomposites and Au-Pd-Pt trimetallic nanotubes are prepared by MW irradiation method. This procedure was carried out in an MW oven (LG Grill, Intellowave, $160-800 \mathrm{~W}$, consumption $800 \mathrm{~W}$, output power $320 \mathrm{~W}$, and frequency $2450 \mathrm{MHz}$ ) operating in a cyclic mode $(\mathrm{ON} 15 \mathrm{~s}$, OFF $5 \mathrm{~s}$ ) to prevent intense boiling of sol as well as aggregation. As synthesized trimetallic nanoparticles were sonicated in a Fast Clean-Ultrasonicator.

2.2.1. Synthesis of Au-Pt-Ag Trimetallic Nanocomposites. Citrate-stabilized Au nanoparticles were prepared according to the reported method by us [16]. Briefly, $10 \mathrm{~mL}$ of aqueous $0.1 \% \mathrm{HAuCl}_{4} \cdot 3 \mathrm{H}_{2} \mathrm{O}$ was heated to a boiling and $2 \mathrm{~mL}$ of $1 \%$ trisodium citrate was then added with stirring. The reaction mixture was heated for $4 \mathrm{~min}(240 \mathrm{sec})$ and cooled to room temperature. The solution was turned to vivid magenta from slight yellow, indicating the formation of $\mathrm{Au}$ nanoparticles. $10 \mathrm{~mL}$ of $0.1 \% \mathrm{H}_{2} \mathrm{PtCl}_{6} \cdot 6 \mathrm{H}_{2} \mathrm{O}$ was then added to the $\mathrm{Au}$ nanoparticles followed by the addition of $2 \mathrm{~mL} 1 \%$ trisodium citrate with stirring. Finally $10 \mathrm{~mL}$ of $0.1 \% \mathrm{AgNO}_{3}$ was added into the $\mathrm{Au} / \mathrm{Pt}$ nanoparticles. The overall reaction time for the preparation of trimetallic nanocomposites are $420 \mathrm{sec}$. Then the prepared sol was sonicated for $1 \mathrm{hr}$.

2.2.2. Synthesis of $A u-P d-P t$ Trimetallic Nanotubes. In atypical synthesis of Pd containing trimetallic nanotubes, $45 \mathrm{~mL}$ of aqueous $1.20 \mathrm{mM} \mathrm{HAuCl} \cdot 3 \mathrm{H}_{2} \mathrm{O}$ was heated to a boil and $2 \mathrm{~mL}$ of $1 \%$ trisodium citrate was then added with stirring. The reaction mixture was heated for $4 \mathrm{~min}$ (240 s) and cooled to room temperature. The solution was turned to vivid magenta from slight yellow, indicating the formation of $\mathrm{Au}$ nanoparticles. $3 \mathrm{~mL}$ of $1 \%$ PVP was then added to the solution and stirred at room temperature overnight. The prepared sol was sonicated for $40 \mathrm{~min}$. Deaerated aqueous $\mathrm{PdCl}_{2}(2.0 \mathrm{~mL}$ of $35 \mathrm{mM})$ was added to PVP-stabilized $\mathrm{Au}$ nanoparticles, with stirring under nitrogen atmosphere
(3 min). After $15 \mathrm{~min} 2.0 \mathrm{~mL}$ of a $1 \%$ trisodium citrate solution was again added dropwise to the solution with constant stirring. The vivid magenta coloured solution turned brown indicating the formation of nano-Pd on the PVPstabilized Au nanoparticles. The stirring was continued for an additional $3 \mathrm{hr}$. Then $2.0 \mathrm{~mL}$ of $20 \mathrm{mM} \mathrm{H}_{2} \mathrm{PtCl}_{6} \cdot 6 \mathrm{H}_{2} \mathrm{O}$ was added and the solution was stirred for another $2 \mathrm{hr}$ at room temperature. Finally the prepared Au-Pd-Pt sol was sonicated for $1 \mathrm{hr}$ to get the fine dispersion of nanoparticles.

2.3. Characterization of Nanoparticles. UV-vis (ultraviolet and visible light) absorbance spectra were measured over a range of 200-800 $\mathrm{nm}$ with a Shimadzu UV-1650PC recording spectrometer using a quartz cell with $10 \mathrm{~mm}$ of optical path length. High-resolution scanning electron microscopy (HRSEM), elementary dispersive X-ray analysis (EDX) experiments were carried out on a FEI Quanta FEG 200 instrument with EDX analysis facility at $25^{\circ} \mathrm{C}$. Wide-angle powder Xray diffraction (XRD) pattern was recorded with an XPERTPRO diffractometer equipped with monochromatic $\mathrm{Cu} \mathrm{K} \alpha$ radiation by using a step scan programme.

\section{Results and Discussion}

The MW irradiation method for the preparation of $\mathrm{Au}$ $\mathrm{Pt}-\mathrm{Ag}$ trimetallic nanocomposites and Au-Pd-Pt trimetallic nanotubes is shown in Figures 1 and 2. From the viewpoint of sequential electron transfer [17], we chose the combination of the trimetals. The ionization potentials of $\mathrm{Au}, \mathrm{Pt}, \mathrm{Ag}$, and $\mathrm{Pd}$ are known to be $9.225,8.620,7.576$, and $8.340 \mathrm{eV}$, respectively. Ionization energy of the corresponding bulk metals is also shown for the comparison in Figure 3 [18].

\subsection{UV-Vis Spectral Studies}

3.1.1. Au-Pt-Ag Trimetallic Nanocomposites. The absorbance spectrum of the sols containing various nanoparticles for the comparison has been given in Figure 4. The absorbance studies have been carried out after ensuring complete reduction of the metal ions based on the kinetics of reduction of individual metal ions. Absorbance spectra corresponding to the individual $\mathrm{Au}, \mathrm{Pt}$, and $\mathrm{Ag}$ nanoparticles are also given 


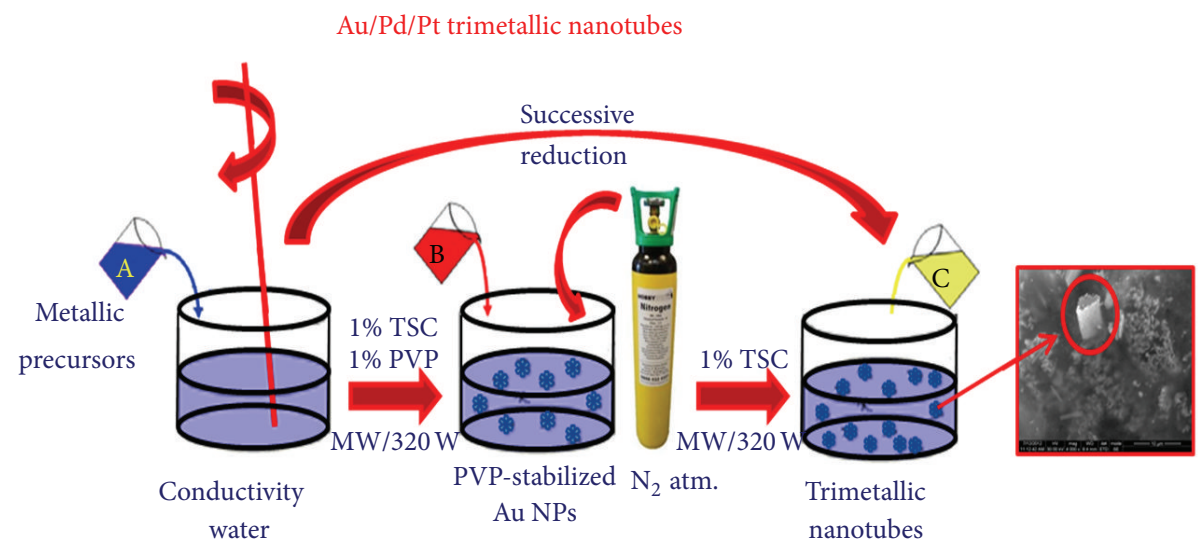

FIgURE 2: MW irradiation method for the preparation of Au-Pd-Pt trimetallic nanotubes.
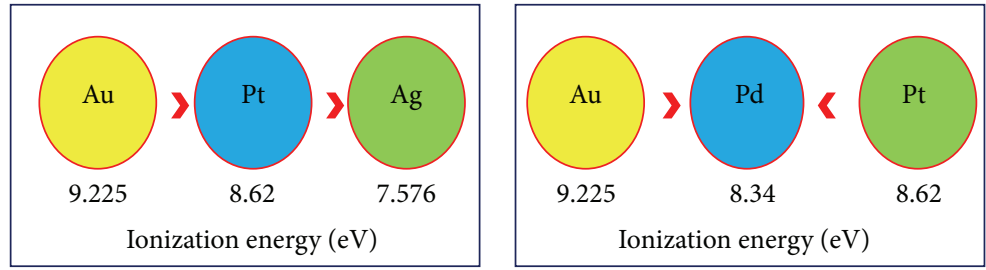

FIGURE 3: Ionization energy of the corresponding bulk metals.

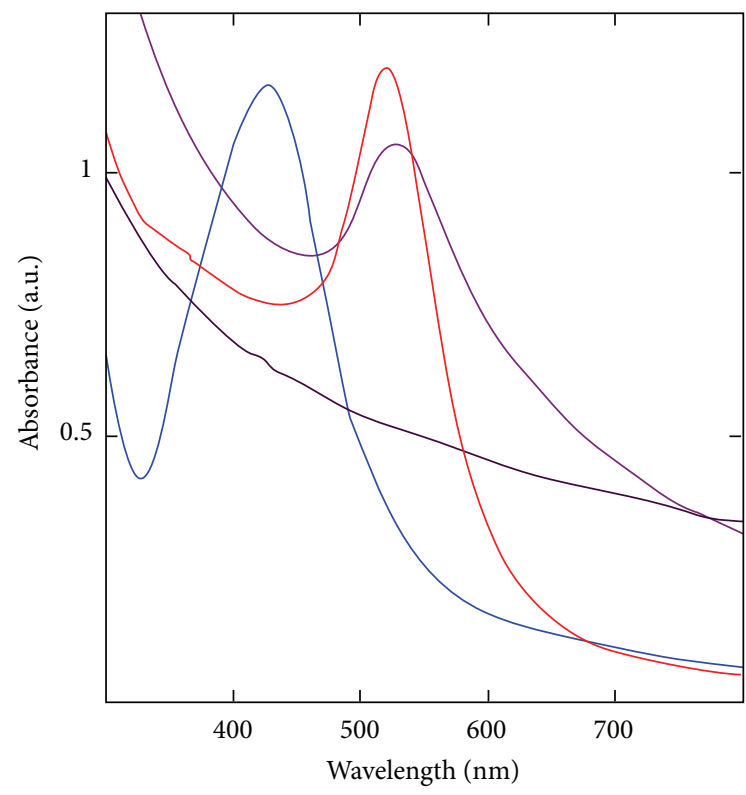

FIGURE 4: UV-vis spectrum of the colloidal dispersion of polymer protected Au nanoparticles.

for comparison. The absorption peak at $530 \mathrm{~nm}$ and $430 \mathrm{~nm}$ can be attributed to the surface plasmon absorption of $\mathrm{Au}$ and $\mathrm{Ag}$ nanoparticles (note that $\mathrm{Pt}$ colloids do not have an individual visible absorbance). The absence of peaks at $310 \mathrm{~nm}$, characteristic of unreduced $\mathrm{Au}$ (III) ion, and peaks at

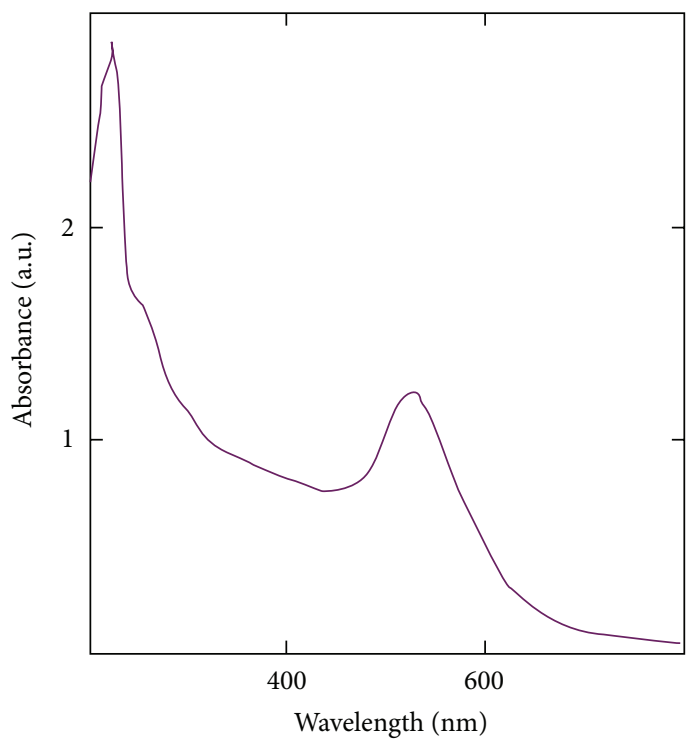

FIGURE 5: UV-vis spectrum of the colloidal dispersion of polymer protected Au nanoparticles.

378, $460 \mathrm{~nm}$ indicates the complete reduction of Pt (IV) ion [19]. But, in the case of Au-Pt-Ag trimetallic nanocomposites, a clear single absorbance at $528 \mathrm{~nm}$ was attributed to the surface plasmon resonance of trimetallic nanoparticles. The absence of multiple bands in the spectrum also rules out the presence of individual $\mathrm{Au}, \mathrm{Pt}$, and $\mathrm{Ag}$ nanoparticles in the dispersion [16]. 


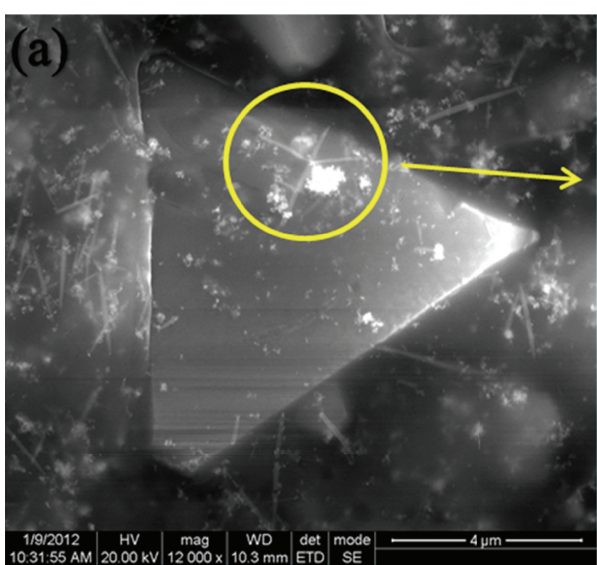

(a)

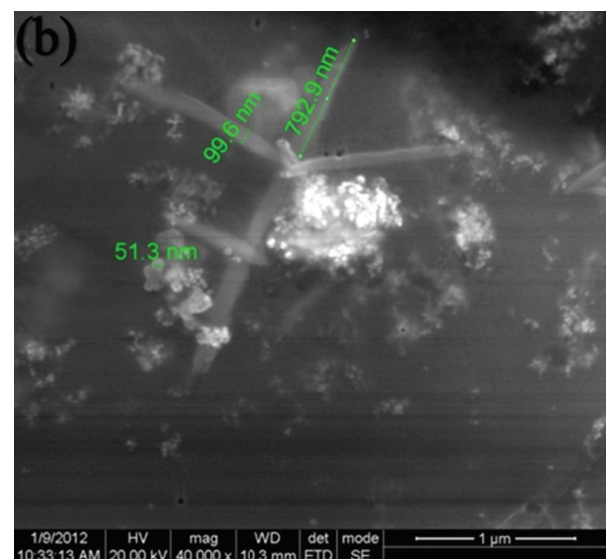

(b)

Figure 6: A typical HR-SEM image of Au-Pt-Ag trimetallic nanocomposites. Magnifications at (a) 12000x (20 kV) and (b) 20000x (20 kV). Scale bars (a) $4 \mu \mathrm{m}$ and (b) $1 \mu \mathrm{m}$.

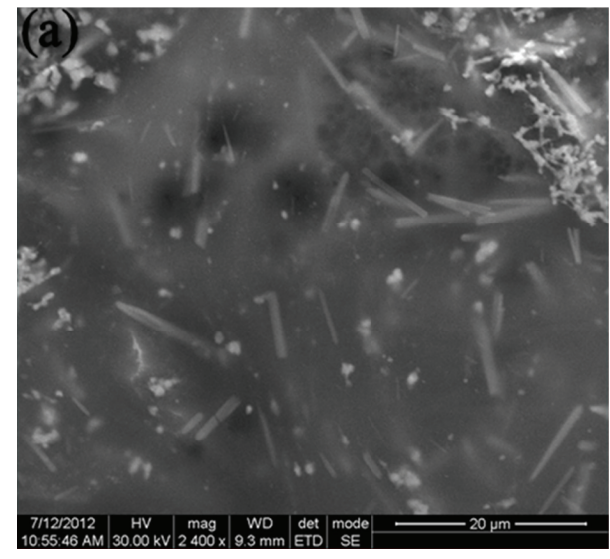

(a)

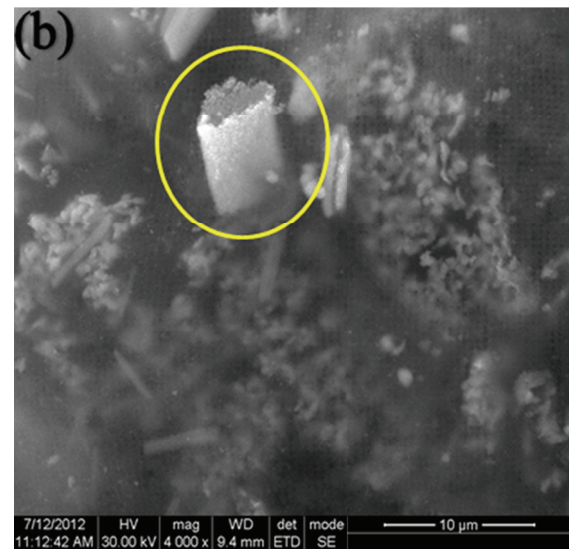

(b)

Figure 7: A typical HR-SEM image of Au-Pd-Pt trimetallic nanotubes. Magnifications at (a) 2400x (30 kV) and (b) $7000 x$ (30 kV). Scale bars (a) $20 \mu \mathrm{m}$ and (b) $10 \mu \mathrm{m}$.

3.1.2. Au-Pd-Pt Trimetallic Nanotubes. Figure 5 shows the UV-Vis spectrum of the colloidal dispersion of polymer protected $\mathrm{Au}$ nanoparticles (note that $\mathrm{Pd}$ and $\mathrm{Pt}$ colloids do not have an individual visible absorbance). A clear surface plasmon absorbance peak which appeared at $528 \mathrm{~nm}$ is corresponding to the presence of $\mathrm{Au}$ nanoparticles and the vivid magenta in colour sol also indicating the presence of Au nanoparticles.

\subsection{High-Resolution Scanning Electron Microscopic (HR-SEM) Studies}

3.2.1. Au-Pt-Ag Trimetallic Nanocomposites. HR-SEM images indicate that the particles are nearly nanowires $(\mathrm{Au})$ and nanocomposite $(\mathrm{Ag}-\mathrm{Pt})$ structure, the length of the nanowire is $792 \mathrm{~nm}$ and the width is 99 and $51 \mathrm{~nm}$ (Figure 6(b)). From Figure 6(a), it is inferred that the nanocomposites are not well dispersed as individual nanoparticles and showed Au nanowires and Ag-Pt bimetallic nanoparticles. The length and width of the nanoparticles (Figure 6(b)) are indicated on the shape of the nanoparticles.

3.2.2. Au-Pd-Pt Trimetallic Nanotubes. Figure 7 shows HRSEM images of as-synthesized Au-Pd-Pt trimetallic nanotubes. HR-SEM investigation clearly reveals that the nanoparticles were uniformly having nanotube shape. The length of the nanotube is $141 \mathrm{~nm}$ (Figure 7(b)) and width of that one is $152 \mathrm{~nm}$.

3.3. Energy Dispersive X-Ray Microanalysis (EDX). One of the most revealing analytical methods for the composition of trimetallic nanoparticle is energy dispersive X-ray spectroscopy (EDX). Figures 8 and 9 show the EDX elemental analysis of as-synthesized $\mathrm{Au}-\mathrm{Pt}-\mathrm{Ag}$ trimetallic nanocomposites and Au-Pd-Pt trimetallic nanotubes. All the $\mathrm{K}$ and $\mathrm{L}$ emission peaks for trimetals are observed. The carbon and copper peaks are due to the HR-SEM holding grid. No other obvious peak attributable to impurity is detected. 


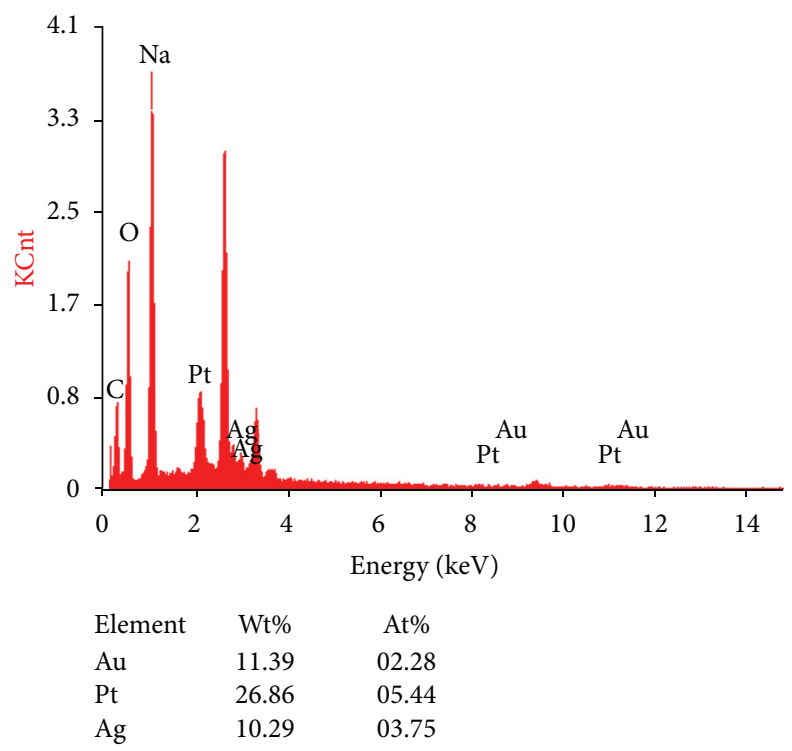

Figure 8: EDX elemental data of Au-Pt-Ag trimetallic nanocomposites.

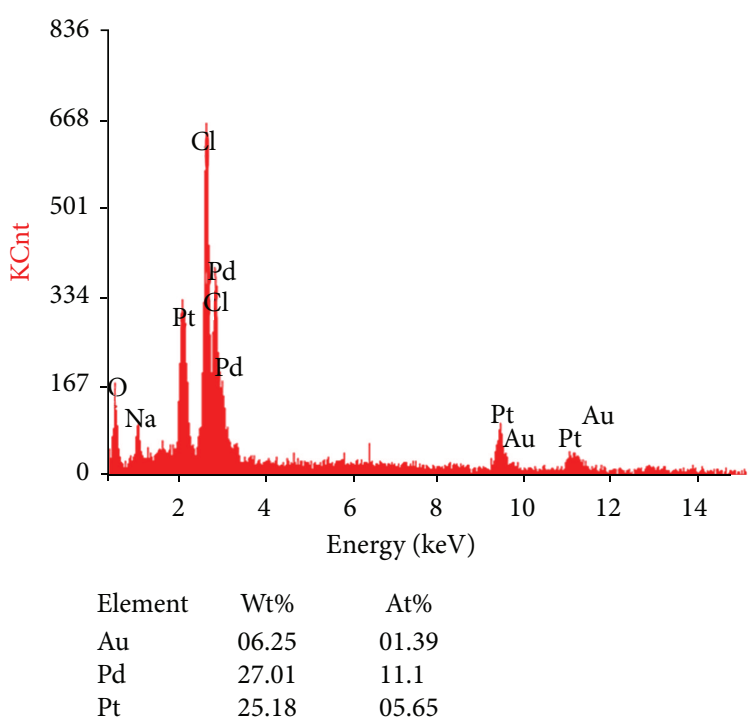

FIgURE 9: EDX elemental data of Au-Pd-Pt trimetallic nanotubes.

This result indicates that as-synthesized product is composed of high purity trimetallic nanoparticles. The carbon and oxygen signals were most likely due to the reducing agents on the surface of the prepared nanoparticles. EDX analysis of Au-Pt-Ag trimetallic nanocomposites gave weight of $11.39 \% \mathrm{Au}, 26.86 \% \mathrm{Pt}$, and $10.23 \% \mathrm{Ag}$ in the sample. It indicated the formation of trimetallic nanocomposites in the ratio of $1: 3: 1$. For Au-Pd-Pt trimetallic nanotubes EDX analysis gave $6.25 \% \mathrm{Au}, 25.18 \% \mathrm{Pd}$, and $27.01 \% \mathrm{Pt}$. It indicated the formation of trimetallic nanotubes in the ratio of $1: 4: 4$. From the above results, the maximum percentage of Pt nanoparticles is present on the surface of Au-Pt-Ag trimetallic nanocomposites and $\mathrm{Pd}$ and $\mathrm{Pt}$ nanoparticles are present on the surface of as-synthesised Au-Pd-Pt trimetallic nanotubes.
3.4. XRD Studies. The diffractograms of the trimetallic combinations generally show broad bands while their monometallic counterparts exhibit fairly sharp bands [19]. The particle sizes of the trimetallic combinations determined from the XRD spectra correlate well with the sizes obtained from HR-SEM measurements. The particle size is calculated based on the Scherrer's equation:

$$
D=\frac{K \lambda}{\beta s \operatorname{Cos} \theta},
$$

where $D$ corresponds to the particle size, $K$ is the shapedependent Scherrer's constant, $\lambda$ is the wavelength of radiation, $\beta s$ is the full peak width at half-maximum (FWHM) of the peak, and $\theta$ is the Bragg diffraction angle [20]. 


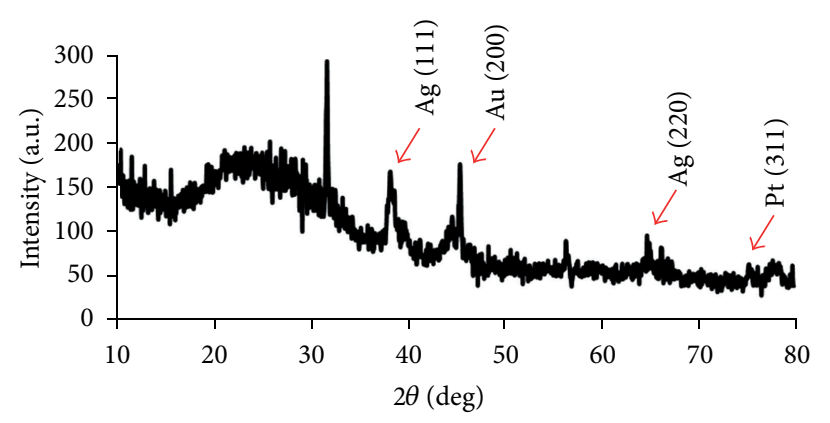

Figure 10: Wide-angle XRD pattern of Au-Pt-Ag trimetallic nanocomposites.

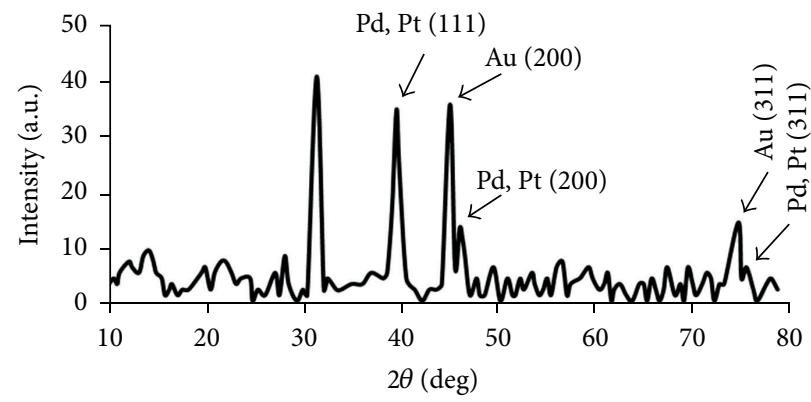

FIgURE 11: Wide-angle XRD pattern of Au-Pd-Pt trimetallic nanotubes.

3.4.1. Au-Pt-Ag Trimetallic Nanocomposites. Figure 10 shows the XRD of Au-Pt-Ag trimetallic nanocomposites. The diffraction patterns indicate a broad diffraction peak $(2 \theta$ about $\left.45.4^{\circ}\right)$ corresponding to $\left(\begin{array}{lll}2 & 0 & 0\end{array}\right)$ reflection. This suggested that the prepared particles have a very small size and a face-centered cubic (fcc) structure. The XRD also indicated the reflection peak that appears at $38.12^{\circ}(2 \theta)$ corresponds to the (lll 111$)$ crystallographic plane and the peak at $64.83^{\circ}(2 \theta)$ corresponds to the (2 20$)$ plane. The diffractograms show that $\left(\begin{array}{lll}3 & 1 & 1\end{array}\right)$ crystallographic surface is marked for Pt $\left(2 \theta=75.29^{\circ}\right)$. The above crystalline data are verified by JCPDS card number of $\mathrm{Au}, \mathrm{Pt}$, and Ag. Table 1 shows the crystalline sizes of nanoparticles and identification of nanoparticles present in trimetallic nanocomposites.

3.4.2. Au-Pd-Pt Trimetallic Nanotubes. Figure 11 shows the $\mathrm{XRD}$ pattern of Au-Pd-Pt trimetallic nanotubes. The wideangle XRD pattern showed randomly oriented fcc crystals. A diffraction peak which appeared at $2 \theta=45.17^{\circ}$ and $75.02^{\circ}$ corresponds to $\mathrm{Au}\left(\begin{array}{lll}2 & 0 & 0\end{array}\right)$ and $\mathrm{Au}\left(\begin{array}{lll}3 & 1 & 1\end{array}\right)$ planes. The XRD also indicated that the reflection peaks that appeared at $2 \theta=$ $39.62^{\circ}, 46.68^{\circ}$, and $75.32^{\circ}$ ascribed to $\mathrm{Pd} / \mathrm{Pt}(111), \mathrm{Pd} / \mathrm{Pt}(20$ $0)$, and $\mathrm{Pd} / \mathrm{Pt}\left(\begin{array}{lll}3 & 1 & 1\end{array}\right)$ planes. Owing to the lattice mismatch factor [21], it was very difficult to resolve the peaks for $\mathrm{Pd}$ and $\mathrm{Pt}$ in the XRD pattern, whereas the peaks of $\mathrm{Au}$ and $\mathrm{Pd} / \mathrm{Pt}$ could be readily distinguished by XRD. Table 2 shows the crystalline sizes of nanoparticles and the identification of nanoparticles present in trimetallic nanotubes. From all of
TABLE 1: The crystalline sizes of nanoparticles and identification of nanoparticles present in $\mathrm{Au}-\mathrm{Pt}-\mathrm{Ag}$ trimetallic nanocomposites.

\begin{tabular}{lcccc}
\hline Sl. no. & 2 Theta $\left({ }^{\circ}\right)$ & $d(\mathrm{~A})$ & FWHM (deg) & $\begin{array}{c}\text { Crystalline } \\
\text { size }(\mathrm{nm})\end{array}$ \\
\hline 1 & 38.12 & 2.27293 & 0.49 & 3.66 \\
2 & 45.40 & 2.00579 & 0.20 & 4.25 \\
3 & 64.83 & 1.94330 & 0.79 & 4.79 \\
4 & 75.29 & 1.26514 & 0.59 & 7.85 \\
\hline
\end{tabular}

TABLE 2: The crystalline sizes of nanoparticles and identification of nanoparticles present in Au-Pd-Pt trimetallic nanotubes.

\begin{tabular}{lcccc}
\hline Sl. no & 2 Theta $\left(^{\circ}\right)$ & $d(\mathrm{~A})$ & FWHM $(\mathrm{deg})$ & $\begin{array}{c}\text { Crystalline } \\
\text { size }(\mathrm{nm})\end{array}$ \\
\hline 1 & 39.62 & 2.27293 & 0.500 & 3.67 \\
2 & 45.17 & 2.00579 & 0.304 & 4.80 \\
3 & 46.68 & 1.94330 & 0.110 & 4.33 \\
4 & 75.02 & 1.26514 & 0.310 & 7.47 \\
\hline
\end{tabular}

the above above observation, $\mathrm{Au}$ in the prepared $\mathrm{Au}-\mathrm{Pd}-\mathrm{Pt}$ trimetallic nanotubes was confirmed as forming inner core.

3.5. Surface Energy. The surface energy of selected 4d and 5d metals is given in Table 3. In addition to that cohesive energy and atomic radius are also tabulated. From this viewpoint, $\mathrm{Pt}$ has more surface energy compared with $\mathrm{Au}, \mathrm{Ag}$, and Pd. So, it is one of the reasons for Pt is present on the surface. 

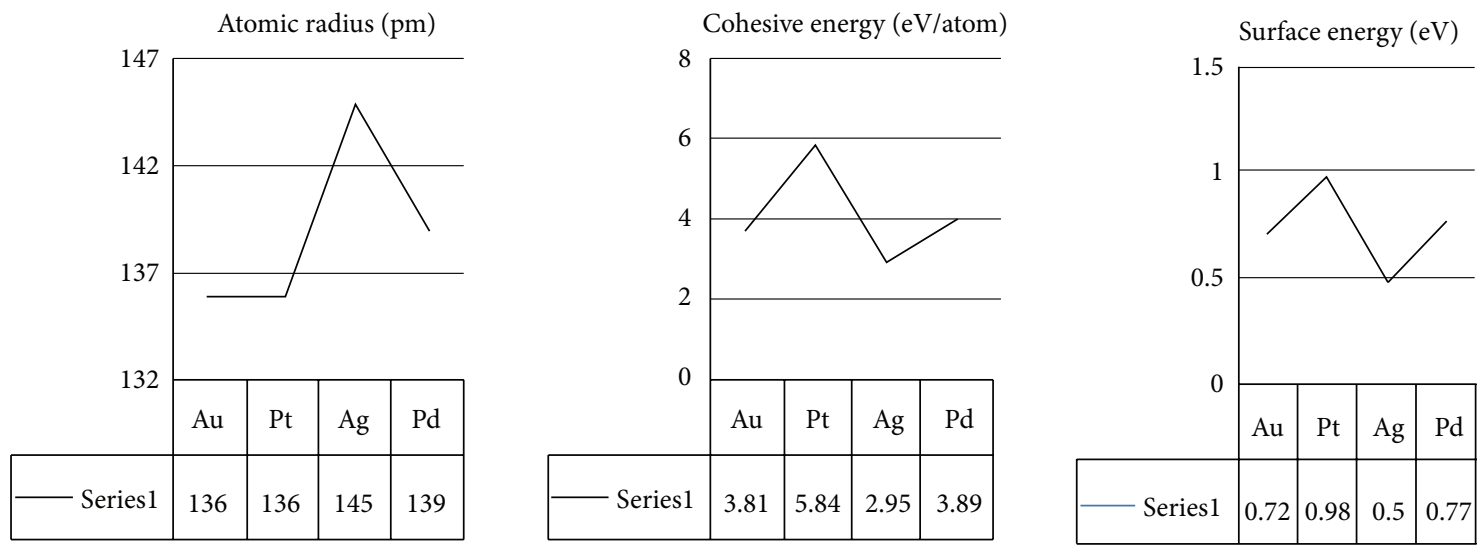

FIgURE 12: Surface energy, cohesive energy, and atomic radius are shown for comparison of $\mathrm{Au}, \mathrm{Pt}, \mathrm{Ag}$, and $\mathrm{Pd}$ metals.

TABle 3: Surface energy, cohesive energy and atomic radius of the $\mathrm{Au}, \mathrm{Pt}, \mathrm{Ag}$ and $\mathrm{Pd}$ metals.

\begin{tabular}{lcccc}
\hline Property & $\mathrm{Au}$ & $\mathrm{Pt}$ & $\mathrm{Ag}$ & $\mathrm{Pd}$ \\
\hline Surface energy & $0.72 \mathrm{eV}$ & $0.98 \mathrm{eV}$ & $0.50 \mathrm{eV}$ & $0.77 \mathrm{eV}$ \\
Cohesive energy & $3.81 \mathrm{eV} /$ atom & $5.84 \mathrm{eV} /$ atom & $2.95 \mathrm{eV} /$ atom & $3.89 \mathrm{eV} /$ atom \\
Atomic radius & $136 \mathrm{pm}$ & $136 \mathrm{pm}$ & $145 \mathrm{pm}$ & $139 \mathrm{pm}$ \\
\hline
\end{tabular}

Surface energy, cohesive energy, and atomic radius are shown for comparison (Figure 12) of $\mathrm{Au}, \mathrm{Pt}, \mathrm{Ag}$, and $\mathrm{Pd}$ metals.

\section{Conclusions}

In conclusion, for the first time, simple MW-irradiated syntheses of $\mathrm{Au}-\mathrm{Pt}-\mathrm{Ag}$ trimetallic nanocomposites and $\mathrm{Au}-$ $\mathrm{Pd}-\mathrm{Pt}$ trimetallic nanotubes are reported. The UV-visible and HR-SEM confirmed the formation of nanocomposites and nanotube-shaped nanoparticles. EDX analysis confirmed that the percentage of $\mathrm{Pt}$ is maximum on the surface of trimetallic nanoparticles and nanotubes. XRD crystal data revealed that the particles are very small in size. The results reported here will inspire the further design of multilayered metallic nanoparticles.

\section{Acknowledgments}

The author (B. Karthikeyan) is highly thankful for UGC, New Delhi, for granting a major research project. Electron microscopy was performed at the DSTUNS and SAIF-IIT Madras. XRD data were recorded at the Department of Physics, Alagappa University, Karaikudi. The coauthor (B. Loganathan) is thankful for UGC for the award of BSR-SAP fellowship.

\section{References}

[1] P. Raveendran, J. Fu, and S. L. Wallen, "Completely "green" synthesis and stabilization of metal nanoparticles," Journal of the American Chemical Society, vol. 125, no. 46, pp.13940-13941, 2003.
[2] M. R. Buck, J. F. Bondi, and R. E. Schaak, "A total-synthesis framework for the construction of high-order colloidal hybrid nanoparticles," Nature Chemistry, vol. 4, no. 1, pp. 37-44, 2012.

[3] C. Xu, J. Xie, D. Ho et al., "Au- $\mathrm{Fe}_{3} \mathrm{O}_{4}$ dumbbell nanoparticles as dual-functional probes," Angewandte Chemie International Edition, vol. 47, pp. 173-176, 2008.

[4] J. Choi, Y. Jun, S. Yeon, H. C. Kim, J. Shin, and J. Cheon, "Biocompatible heterostructured nanoparticles for multimodal biological detection," Journal of the American Chemical Society, vol. 128, no. 50, pp. 15982-15983, 2006.

[5] C. Xu, B. Wang, and S. Sun, "Dumbbell-like au- $\mathrm{fe}_{3} \mathrm{O}_{4}$ nanoparticles for target-specific platin delivery," Journal of the American Chemical Society, vol. 131, no. 12, pp. 4216-4217, 2009.

[6] H. Yin, C. Wang, H. Zhu, S. H. Overbury, S. Sun, and S. Dai, "Colloidal deposition synthesis of supported gold nanocatalysts based on $\mathrm{Au}-\mathrm{Fe}_{3} \mathrm{O}_{4}$ dumbbell nanoparticles," Chemical Communications, no. 36, pp. 4357-4359, 2008.

[7] T. Mokari, E. Rothenberg, I. Popov, R. Costi, and U. Banin, "Selective growth of metal tips onto semiconductor quantum rods and tetrapods," Science, vol. 304, no. 5678, pp. 1787-1790, 2004.

[8] B. Karthikeyan and M. Murugavelu, "Nano bimetallic Ag/Pt system as efficient opto and electrochemical sensing platform towards adenine," Sensors and Actuators B: Chemical, vol. 163, no. 1, pp. 216-223, 2012.

[9] S. Patra and H. Yang, "Synthesis of trimetallic Au@Pb@Pt coreshell nanoparticles and their electrocatalytic activity toward formic acid and methanol," Bulletin of the Korean Chemical Society, vol. 30, no. 7, pp. 1485-1488, 2009.

[10] H. Zhang, M. Okumura, and N. Toshima, "Stable dispersions of PVP-protected $\mathrm{Au} / \mathrm{Pt} / \mathrm{Ag}$ trimetallic nanoparticles as highly active colloidal catalysts for aerobic glucose oxidation," Journal of Physical Chemistry C, vol. 115, no. 30, pp. 14883-14891, 2011. 
[11] P. Venkatesan and J. Santhanalakshmi, "Core-shell bimetallic Au-Pd nanoparticles: synthesis, structure, optical and catalytic properties," Journal of Nanoscience and Nanotechnology, vol. 1, pp. 43-47, 2011.

[12] B. N. Wanjala, J. Luo, R. Loukrakpam et al., "Nanoscale alloying, phase-segregation, and core-shell evolution of gold-platinum nanoparticles and their electrocatalytic effect on oxygen reduction reaction," Chemistry of Materials, vol. 22, no. 14, pp. 42824294, 2010.

[13] L. Wang and Y. Yamauchi, "Strategic synthesis of trimetallic Au@Pd@Pt core-shell nanoparticles from poly(vinylpyrrolidone)-based aqueous solution toward highly active electrocatalysts," Chemistry of Materials, vol. 23, no. 9, pp. 2457-2465, 2011.

[14] P. Fang, S. Duan, X. Lin et al., "Tailoring Au-core Pd-shell Pt-cluster nanoparticles for enhanced electrocatalytic activity," Chemical Science, vol. 2, no. 3, pp. 531-539, 2011.

[15] H. Yin, T. Yamamoto, Y. Wada, and S. Yanagida, "Largescale and size-controlled synthesis of silver nanoparticles under microwave irradiation," Materials Chemistry and Physics, vol. 83, no. 1, pp. 66-70, 2004.

[16] B. Karthikeyan and B. Loganathan, "Strategic green synthesis and characterization of $\mathrm{Au} / \mathrm{Pt} / \mathrm{Ag}$ trimetallic nanocomposites," Materials Letters, vol. 85, pp. 53-56, 2012.

[17] N. Toshima, Macromolecular Complexes: Dynamic Interactions and Electronic Processes, edited by E. Tsuchida, Japan Scientific Societies Press, Tokyo, Japan, 1991.

[18] N. Toshima, "Polymer-assisted composites of trimetallic nanoparticles with a three-layered core-shell structure for catalyses," Nanohybridization of Organic-Inorganic Materials. Advances in Matetrials Research, vol. 13, pp. 55-79, 2009.

[19] T. Yonezawa and N. Toshima, "Mechanistic consideration of formation of polymer-protected nanoscopic bimetallic clusters," Journal of the Chemical Society, Faraday Transactions, vol. 91, no. 22, pp. 4111-4119, 1995.

[20] D. S. Sheny, J. Mathew, and D. Philip, "Phytosynthesis of Au, $\mathrm{Ag}$ and $\mathrm{Au}-\mathrm{Ag}$ bimetallic nanoparticles using aqueous extract and dried leaf of Anacardium occidentale," Spectrochimica Acta A: Molecular and Biomolecular Spectroscopy, vol. 79, no. 1, pp. 254-262, 2011.

[21] S. E. Habas, H. Lee, V. Radmilovic, G. A. Somorjai, and P. Yang, "Shaping binary metal nanocrystals through epitaxial seeded growth,” Nature Materials, vol. 6, no. 9, pp. 692-697, 2007. 

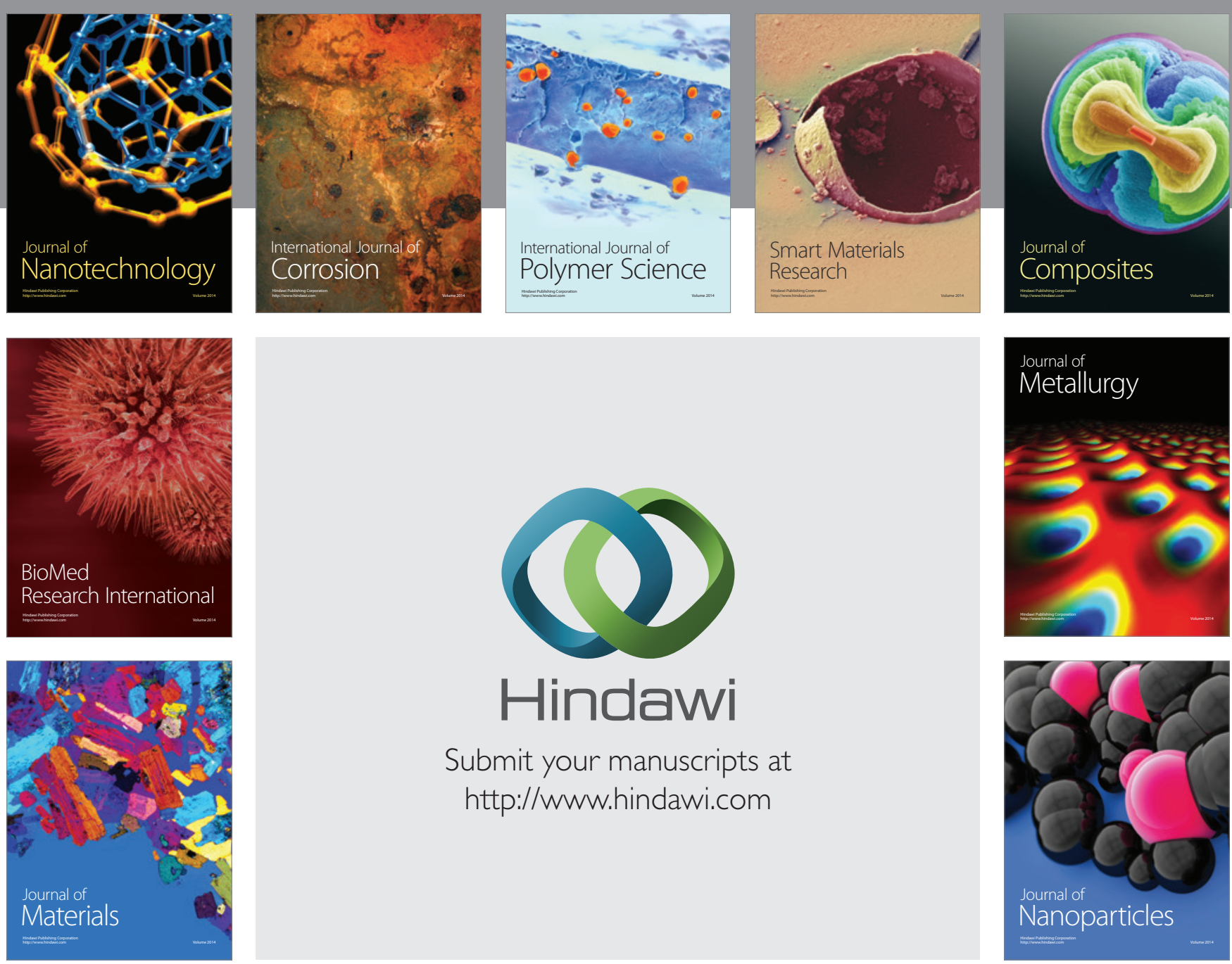

Submit your manuscripts at http://www.hindawi.com
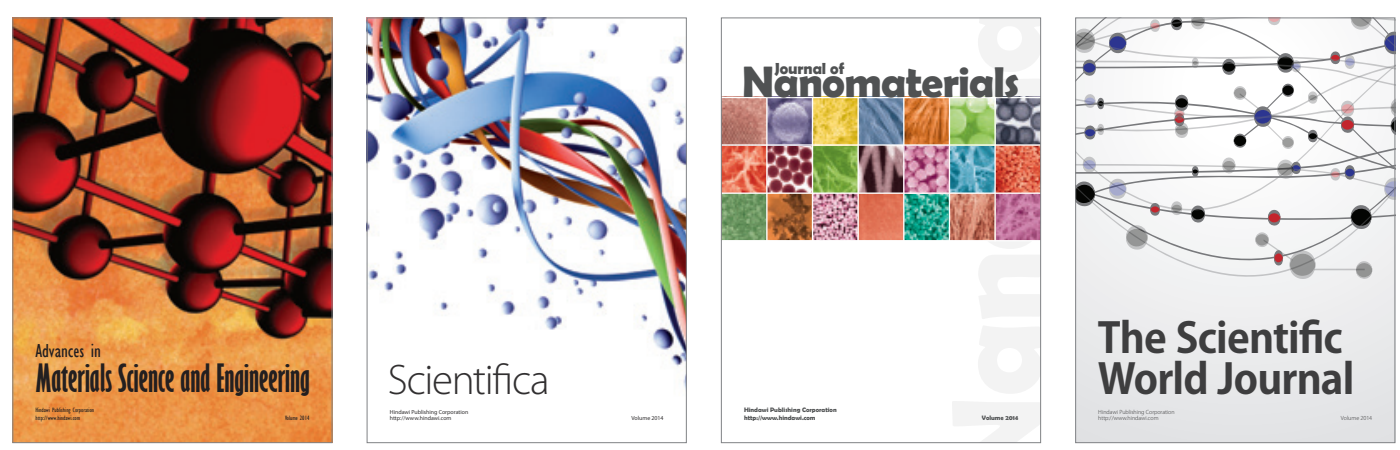

\section{The Scientific World Journal}
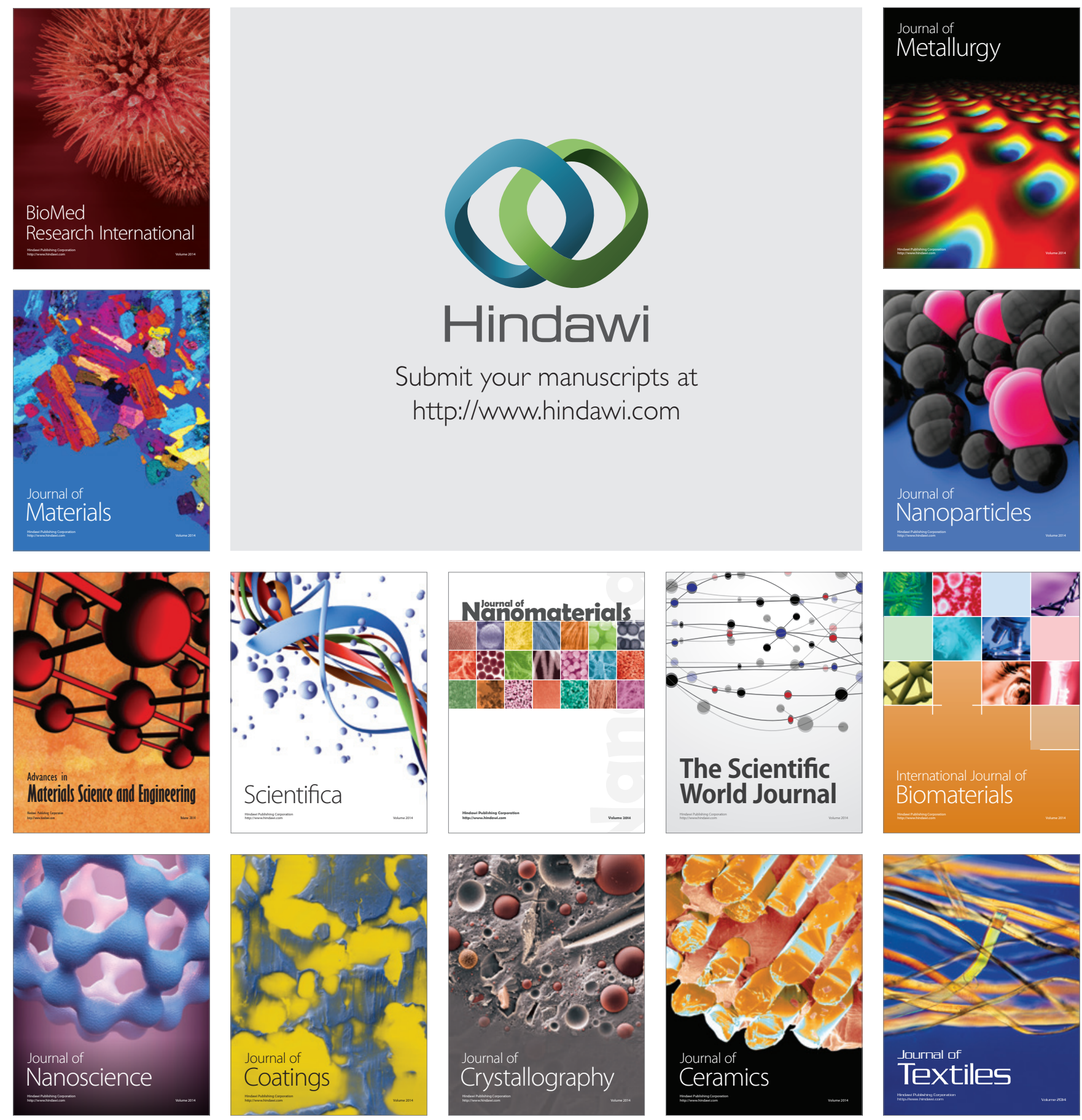